\title{
A novel k-Combination First-Fit Algorithm for Three-dimensional Knapsack Packing Problem
}

\author{
Xiaoyu Huang, Mingyao Qi, Ying Zhang, Shulin Zhao \\ Division of Logistics and Transportation, Graduate School at Shenzhen, Tsinghua University, Shenzhen, 518055, China
}

\begin{abstract}
A novel k-Combination First-Fit Algorithm is presented in this paper, which provides a high-speed and high-accuracy solution for Three-dimensional Knapsack Packing Problem (3DKP). Previously, limited solutions for 3DKP exist because 3DKP $\in N P$, which indicates there is no polynomial time exact algorithm. Considered both approximate performance and time cost, the objective of the present study is to design and analyze a solution for 3DKP in order to obtain desired performance within limited time. Outer k-Combination Algorithm is introduced for facilitating the detection and reduction of time cost, meanwhile, Inner Greedy-First-Fit Packing Algorithm is implemented for obtaining desired packing performance. In stark contrast, the experimental results demonstrate that approximate performance of the present method is $1.51 \%$ better than the latest 3DKP solution within $\mathbf{5 8 . 3 1 \%}$ less time cost. Therefore, the present solution is more accurate and lower time-cost than other existing approaches, since it has taken both approximate performance and time complexity into consideration. Although the quantitative contribution of it is unknown, there is no doubt that the present method for 3DKP would be extremely advantageous for improvement in 3DKP field.
\end{abstract}

Keywords-multi-objective; bilevel programming; genetic algorithm; interpolation.

\section{INTRODUCTION}

Assume that we are given a set of $n$ rectangular-shaped items, each characterized by length $l_{i}$, width $W_{i}$, height hi and profit $\mathrm{p}_{i}(i=1,2, \ldots, n)$, as well as a three-imensional container (bin) having length $L$, width $\mathrm{W}$ and height $H$. The maximum profit three $\square$ dimensional knapsack (3DKP) problem consists of orthogonally packing a subset of the cubes into he larger cube such that the associated profit sum is maximized. We assume that the items may be rotated, i.e., that they are packed with each edge parallel to arbitrary edges of bin. No further restriction is present, except that all coefficients are assumed to be nonnegative real numbers. Therefore, a packing mode of items in the container is easible if no two items overlap, and if no part of any item exceeds the container. The key idea of our work is to solve 3DKP problem using the proposed k-Combination Greedy-First-Fit Algorithm, where both time complexity and approximate performance are considered.

The 3DKP problem has direct applications in public logistics fields where the objective is to obtain the special optimal selection. Consequently, the interest to study this problem has significantly grown in the last years, with the two appearance of proposals based on different technologies that include Nash genetic algorithm and heuristic approaches. However, one of the most remarkable features of the 3DKP problem is that it is strongly NP-hard because it is a generalization of the well-known one-dimensional knapsack packing problem (1D-KPP), in which a set of $n$ positive weights $\mathrm{Wi}$ has to be optimally considered so that the total weight in the selected subset of items does not exceed a given bin capacity $W$, meanwhile, the total profit is maximized. The conclusion of 1D-KPP is NP-hard is proved in this paper later. Because $1 \mathrm{D}-\mathrm{KPP}$ is the special case of 3DKP arising when $\mathrm{h}_{i}=H$ and $\mathrm{d}_{i}=D$ for all $i=1,2, \ldots, n$, it is self-evident that 3DKP is strongly NP-hard. This fact makes it is extremely difficult to obtain optimal solves for 3DKP problem. In order to solve this issue, a parallel genetic algorithm based on the Island Model and the Nash equilibrium theory for solving the 3DKP problem assuming both, orthogonally packing and rotation prevention is proposed by Daniel Soto et. al [1]. Also, a new iterative heuristic for the two- and three-dimensional knapsack packing problem based on the sequence pair representation [2] using a semi-normalized packing algorithm [3] is developed by Jens Egeblad et. al [4]. It is clear that time complexity and approximation performance is a pair of contradictions for NP-hard problems. Although the previous methods perform acceptably in some cases, however, to our knowledge, they have not considered the fundamental contradiction between time complexity and approximation performance, and they have only focused on approximation performance, which makes these methods have poor practicability.

Details of modeling of the proposed algorithm are proposed in Section 4. The better approximation performance with smaller time complexity is proved by the derivation process described in Section 5, simultaneously, the results demonstrated in Section 5 provide further support for the idea that the kC-FF Algorithm performs well in both time complexity and approximation performance.

\section{DISCUSSED PROBLEMS}

The three-dimensional knapsack problem can be formulated as follows: 


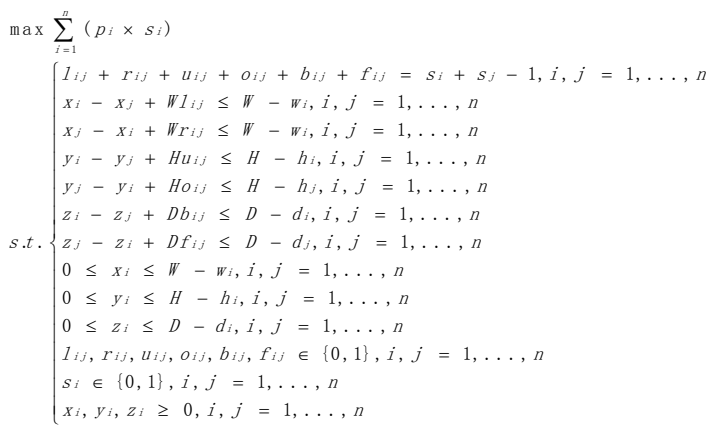

where $W, H, D$ are three dimensions of the knapsack bin, $S_{i}$ is the decision variable indicating whether item $i$ is packed within the knapsack bin, and $l_{i j}, r_{i j}, u_{i j}, o_{i j}, b_{i j}, f_{i j}$ are the binary decision variables indicating the relative position of items $i, j$ where $i<j$.

\section{MODEL AND METHOD}

\section{A. Introduction of approximation ratio}

There is one important concept [4] has failed to notice: Approximation Ratio. Given that $\pi$ is one minimal optimization problem (MIOP) or maximal optimization problem (MAOP), and $I$ is one arbitrary instance of $\pi$. Assume there is an approximation algorithm A for solving Problem $\pi$, and the optimal solution is OPT, the approximation ratio for solving Instance $I$ in Problem $\pi$ is defined in Equation 2:

$$
\left\{\begin{array}{l}
R_{A}(I)=\frac{A(I)}{O P T(I)}, \text { if } \pi \in M I O P \\
R_{A}(I)=\frac{O P T(I)}{A(I)}, \text { if } \pi \in M A O P
\end{array}\right.
$$

\section{B. Introduction of k-Combination-Set}

As mentioned above, time complexity and approximation performance is a pair of contradictions for NP-hard problems. This conclusion is also suitable for 3DKP. Given $\mathrm{n}$ items, we have to iterate for $n$ ! times in order to obtain the optimal solution. Due to the fact that $n$ ! expands at an exponential rate, this problem is difficult to solve. And the main idea to solve this issue is scaling down the number of combinations. Based on this background, we introduce k-Combination-Set to indicate how many items are chosen to be packed first as a set together. Experiments results demonstrate the idea of k-Combination-Set performs well within acceptable time.

\section{Abstract model.}

In order to take both time complexity and approximation performance into consideration, a novel k-Combination
First-Fit Algorithm (kC-FF) for three-dimensional knapsack packing problem is proposed in this paper. Based on the fact that classical methods for optimally solving NP-hard problems result in exponential computational complexities, the key idea of this work is "Divide and Conquer". First, we divide the 3DKP problem into two parts:

(1) Outer: choosing a $\mathrm{k} \square$ Combination subset whose size is $\mathrm{k}$ from the whole set of item.

(2) Inner: packing each $k \square$ Combination subset using Greedy-First-Fit Algorithm.

Second, we conquer this problem by combining and analyzing all the obtained solutions. Experimental results prove that this "Divide and Conquer" strategy is suitable for 3DKP problem, which has the potential to bring significant breakthrough into the loading field.

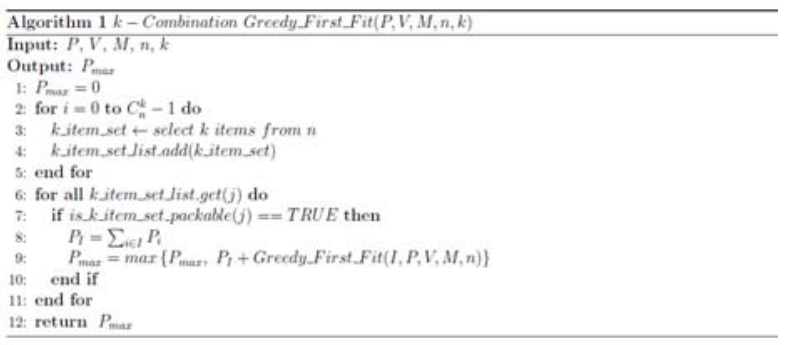

\section{GENETIC ALGORITHM}

According to the idea of k-Combination-Set, we introduce k-Combination Greedy-First-Fit Algorithm 1, which includes a subalgorithm Greedy-First-Fit Algorithm 2. In Algorithm 1, inputs are defined as follows: $\mathrm{P}$ is the value vector of items set, $\mathrm{V}$ is the volume vector of items set, $\mathrm{M}$ is the volume of the knapsack bin, $n$ is the number of all the items, and $\mathrm{k}$ is the number of the items in k-Combination-Set. And Algorithm output the maximal value based on the given $k$. First, we storages all the combinations of k_item_set into k_item_set_list. Second, we traverse all the $\mathrm{k}$ item set to judge whether this set can be packed, and if it is labeled packable, then pack this items set and get the sum of the set's value $P_{I}$. Third, pack the remaining items using Greedy First Fit Algorithm described in Algorithm 2. In Algorithm 2, I is the k_item_set chosen in Algorithm 1, and after sorting by $\frac{P}{V}$, we just pack all the remaining items one by one, and discard the items can not be packed. We adopt the corner-point packing approach described in [5] to pack an item into the bin. Algorithm 2 returns the value sum $\mathrm{S}$ of the remaining items which can be packed. Forth, we update the maximal value sum $P_{I}+S$, and return this maximal value sum when the traversal is over eventually. 


Algorithm 2 Grecdy_First Fit $(I, P, V, M, n)$
Input: $I, P, V, M, n$,
Output: $S$
1: $S_{l}=0$
2. $T=M-\sum_{i \in l} V_{i}$
3. sorting $\Rightarrow T_{i} \geq B_{i} \geq \ldots \geq V_{i}$
4. for $i=0$ to $n$ do
5. if $(i \notin I)$ and $\left(V_{i} \leq T\right)$ then
6. $\quad S S_{i}=S_{i}+P_{i}$
7: $\quad T=T-V_{i}$
8. end if
9. end for
10: $S=\max \left\{S_{l}, \max \left\{P_{i} \mid i \notin I\right\}\right\}$
11: return $S$

In order to illustrate time-dependent properties of the present algorithm, time complexity is derived as follows. Lines $2 \square 5$ and Line 6 in Algorithm 1 need O(nk) time-cost, is_k_item_set_packable() in Line 7 is based on the Key-Point Packing Theory described in [6], whose time complexity is $k \times 7 \times n$ if rotation is not allowed, and $k \times 7 \times n \times 6$ when rotation. Therefore, Line 7 needs $\mathrm{O}(\mathrm{n})$ time-cost with or without rotation. In Algorithm 2, time complexity of Line 3 is $\mathrm{O}(\mathrm{n}(\log n))$. As mentioned above, Line 6 using Key-Point Packing Theory needs O(n) time-cost, so time complexity of Line $4 \square 9$ is $\mathrm{O}$ (n2), which indicates that time complexity of Algorithm 2 is $\mathrm{O}(\mathrm{n}(\operatorname{logn})+$ n2) $=\mathrm{O}(\mathrm{n} 2)$. Therefore, Line 9 in Algorithm 1 needs O(n2) time-cost. To sum up, time complexity of the present $\mathrm{k}$-Combination Greedy-First-Fit Algorithm is $\mathrm{O}(\mathrm{nk}+\mathrm{nk} \times \mathrm{c} 1$ $\times \mathrm{n} \times \mathrm{c} 2 \times \mathrm{n} 2)=\mathrm{O}(\mathrm{nk}+3)$, where $\mathrm{c} 1$ and $\mathrm{c} 2$ are constant.

\section{COMPUTATIONAL EXAMPLES AND ANALYSIS}

To the best of our knowledge, there are two main methods for solving three-dimensional knapsack problem. One is Greedy Heuristic Algorithm (GHA) described in [7], which has been widely applied to industrial application due to its rapidity. And another one is the Iterative Heuristic Algorithm based on the Sequence Pair (IHASP) represented in [4], which made a breakthrough in approximation performance of 3DKP problem. In order to illustrate cost/performance comparisons between the present k-Combination First-Fit Algorithm and several previous methods, we have compared total value and time cost of kCFFA with GHA and IHASP.

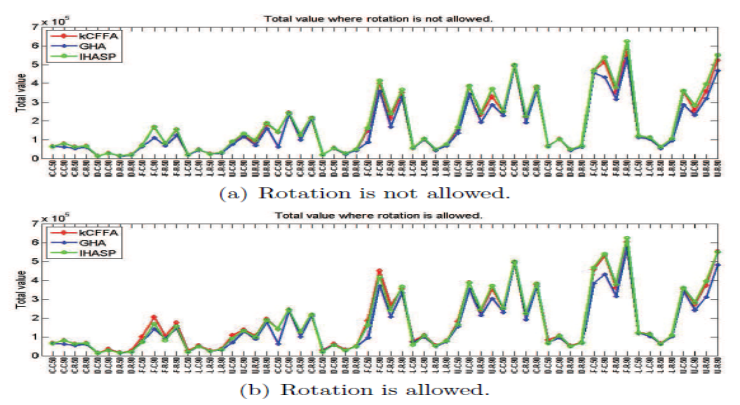

Figure 1. Total value where rotation is and is not allowed are both considered.

From Fig. 1(a), the average total value of kCFFA is 169891.97, GHA is 151832.43, while IHASP is 177420.95 .
One of the authentic reasons why total value of kCFFA is $4.24 \%$ less than IHASP is the restricted constraint that rotation is not allowed has limited the actuating range of kCFFA. Fig. 1(b) illustrates the situation when rotation is allowed. Now the average of kCFFA is 180093.87 , GHA is 157689.00, while IHASP is 177420.95. Taken together, these results have compellingly demonstrated that the appropriate performance of three-dimensional knapsack problem is greatly influenced by the condition whether rotation is allowed or not.
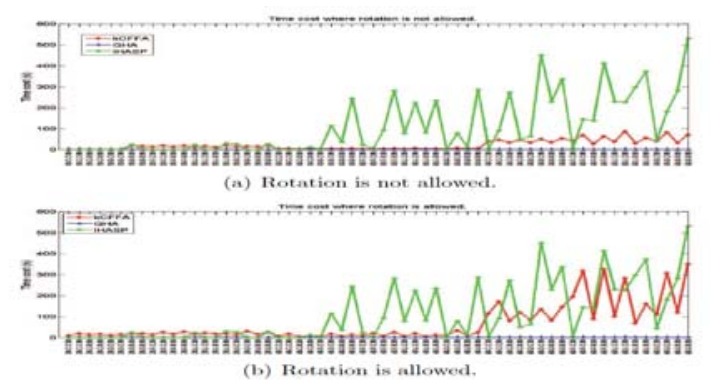

Figure 2. Time cost where rotation is and is not allowed are both considered.

Fig. 2(a) shows that time costs of all item types using GHA are within $0.01 \mathrm{~s}$, while kCFFA is fluctuating from $0.01 \mathrm{~s}$ to $86.48 \mathrm{~s}$, and IHASP is from $0.01 \mathrm{~s}$ to $531.62 \mathrm{~s}$. Under the condition that rotation is not allowed, the average time cost of GHA is $0.01 \mathrm{~s}$, GHA is 21.21s, and IHASP is 105.02s. Figure 10(b) illustrates that the average time cost of kCFFA is $66.34 \mathrm{~s}$, while GHA is $0.01 \mathrm{~s}$, and IHASP is $105.02 \mathrm{~s}$.

\section{CONCLUSIONS}

A novel k-Combination First-Fit Algorithm is presented in this paper, which provides a high-speed and high accuracy solution for Three-dimensional Knapsack Packing Problem (3DKP). In order to take both approximate performance and time cost into consideration, Outer k-Combination Algorithm is introduced for facilitating the detection and reduction of time cost, meanwhile, Inner Key-Point Packing Algorithm is implemented for obtaining desired packing performance. We have compared the present method with other existing approaches in three aspects: total value, percentage of upper bound and time cost. Experimental results reveal that approximate performance of the present method is $1.51 \%$ better than the latest 3DKP solution within $58.31 \%$ less time cost, which proves that the present method for 3DKP is more accurate and lower time-cost than other existing approaches.

\section{ACKNOWLEDGEMENTS}

This work is partially supported by the National Natural Science Foundation of China (71272030), as well as Science \& Technology Foundation of Shenzhen City (CXZZ20130321145336439).

\section{REFERENCES}

[1] D. Soto, W. Soto, Y. Pinz'on, A parallel nash genetic algorithm for the 3d orthogonal knapsack problem 
[2] H. Murata, K. Fujiyoshi, S. Nakatake, Y. Kajitani, Vlsi module placement based on rectangle-packing by the sequence-pair, Computer-Aided Design of Integrated Circuits and Systems, IEEE Transactions on 15 (12) (1996) 1518-1524.

[3] D. Pisinger, Denser packings obtained in o ( $\mathrm{n} \log \log \mathrm{n})$ time, INFORMS Journal on Computing 19 (3) (2007) 395-405.

[4] J. Egeblad, D. Pisinger, Heuristic approaches for the two-and three-dimensional knapsack packing problem, Computers \& Operations Research 36 (4) (2009) 1026-1049.
[5] C. D. Tarantilis, E. E. Zachariadis, C. T. Kiranoudis, A hybrid metaheuristic algorithm for the integrated vehicle routing and three-dimensional container-loading problem, Intelligent Transportation Systems, IEEE Transactions on 10 (2) (2009) 255-271.

[6] S. Martello, D. Pisinger, D. Vigo, The three-dimensional bin packing problem, Operations Research 48 (2) (2000) 256-267 